
allemande

46-1 | 2014

Les fondements normatifs de l'État constitutionnel moderne en Allemagne. Une approche pluridisciplinaire

\title{
Le protestantisme allemand et la création de la République fédérale d'Allemagne (1945-1949)
}

\section{Henri Haller}

\section{(QpenEdition \\ Journals}

Édition électronique

URL : https://journals.openedition.org/allemagne/1244

DOI : $10.4000 /$ allemagne. 1244

ISSN : 2605-7913

Éditeur

Société d'études allemandes

Édition imprimée

Date de publication : 30 juin 2014

Pagination : $35-50$

ISSN : 0035-0974

Référence électronique

Henri Haller, «Le protestantisme allemand et la création de la République fédérale d'Allemagne

(1945-1949) », Revue d'Allemagne et des pays de langue allemande [En ligne], 46-1 | 2014, mis en ligne le 29 juillet 2019, consulté le 18 mai 2021. URL : http://journals.openedition.org/allemagne/1244 ; DOI : https://doi.org/10.4000/allemagne.1244 


\title{
Le protestantisme allemand et la création de la République fédérale d'Allemagne (1945-1949)
}

\author{
- Henri Haller*
}

Le 8 mai 1945, l'armée allemande capitule sans condition. Moins d'un mois plus tard, le 5 juin, les quatre gouvernements alliés annoncent qu'ils prennent en charge la direction de l'Allemagne. Ils adoptent une décision singulière, celle de maintenir théoriquement l'existence de l'Allemagne mais d'en assurer eux-mêmes la souveraineté interne et externe. Ce même jour, une seconde déclaration ramène l'Allemagne à ses frontières du 31 décembre 1937.

Le 8 mai 1945, un pasteur de Düsseldorf, Joachim Beckmann, adresse une requête aux autorités militaires britanniques: il leur demande l'autorisation de se déplacer à bicyclette dans un rayon de $40 \mathrm{~km}$ pour ramener à Essen et à Wuppertal deux autres dirigeants de l'Église confessante et justifie ainsi sa requête: "L'Église confessante a beaucoup souffert et doit être reconstruite dans les meilleurs délais. »

L'objet de notre contribution n'est pas d'envisager l'importance du protestantisme au sein des associations caritatives et des partis politiques - notamment la CDU, parti chrétien à vocation bi-confessionnelle - qui se constituent avant la création de la République fédérale d'Allemagne. Notre but est plutôt de montrer comment, à partir des leçons qu'ils tiraient du passé et de la lutte de l'Église confessante contre le totalitarisme nazi, les protestants allemands, pasteurs comme laïcs engagés, ont tenté de créer une Église nouvelle, et comment, épaulés par les Alliés occidentaux, ils ont pu retrouver une place dans les instances internationales du protestantisme. Le débat central, difficile mais courageux, sur la culpabilité des Églises et du peuple allemand a pu forger pour le protestantisme allemand une nouvelle identité et de nouvelles perspectives. Une dernière partie envisage dans quelle mesure la nouvelle constitution a répondu ou non à ces attentes du protestantisme allemand. Le 23 mai 1949, la Loi fondamentale (Grundgesetz) instaure un statut des Églises qui légitime un certain nombre des prérogatives acquises antérieurement par celles-ci, mais elle a recours aux termes de la Constitution de Weimar pour limiter les compétences des Églises par rapport à l'État.

* Maître de conférences honoraire en études germaniques, Université de Paris Ouest Nanterre La Défense. 


\section{Les pesanteurs historiques du protestantisme allemand}

Si le Kulturkampf a contribué à déterminer la stratégie et l'organisation du catholicisme politique, s'il a créé des structures de résistance et de combat contre l'Allemagne bismarckienne protestante, le Kirchenkampf pour sa part est une phase essentielle et une référence pour comprendre la réorientation et les mutations du protestantisme en Allemagne depuis 1933 et ses conséquences sont manifestes dans l'histoire de l'Allemagne d'après-guerre. Le terme de Kirchenkampf apparaît à la fin de 1933 pour désigner le conflit alors en cours qui portait sur la réorganisation des Églises protestantes allemandes. À partir de 1935, son sens s'élargit à l'ensemble des questions politiques et juridiques concernant la place des Églises protestantes dans l'État nazi. On l'utilise même au sens d'une contestation fondamentale de l'idéologie du nazisme, comprise comme «religion politique» (G. Jacob, décembre 1936). Aujourd'hui, on entend par ce terme l'ensemble de la période de l'histoire des Églises allemandes entre 1933 et 1947. Pour comprendre les enjeux de ce conflit et ses répercussions sur le protestantisme allemand, il convient d'abord de suivre les principales étapes de cet affrontement.

Dans les mois qui ont suivi la prise de pouvoir de Hitler, la politique des nazis vis-àvis des Églises protestantes est dictée par la volonté d'une unification de ces Églises au sein d'une Reichskirche. Cette Reichskirche, sorte d'Église nationale, devait regrouper toutes les Églises protestantes du Reich. Ce plan devait permettre de trouver une solution homogène aux rapports des Églises territoriales avec le nouvel État allemand. S’il y a accord sur le but, les modalités institutionnelles vont faire l'objet de controverses. Hitler s'appuie sur le mouvement des chrétiens allemands ("Glaubensbewegung Deutsche Christen »), fondé en février 1932 en vue des élections ecclésiastiques de Prusse de novembre 1932. En avril 1933, les chrétiens allemands proposent comme candidat au poste d'évêque du Reich (Reichsbischof) Ludwig Müller, aumônier militaire de Königsberg. Hitler le désigne pour être son homme de confiance afin de conduire cette politique d'unification. Les chrétiens allemands s'alignent sur la politique raciste du régime nazi en demandant que soit appliqué dans les Églises protestantes le paragraphe " aryen ", le passage de la loi sur le rétablissement d'une fonction publique dite " nationale », c'est-à-dire excluant les Juifs.

Contre ces exigences se crée le mouvement des « jeunes réformateurs », il regroupe avant tout des théologiens luthériens conservateurs comme Hans Lilje et Martin Niemöller. Bien que soutenant politiquement le nouveau régime, ils refusent que la politique ecclésiastique soit inspirée d'un modèle politique (Führerstaat) et exigent une refonte des institutions partant de ce qui fait l'essence de l'Église. En mai-juin 1933, le gouvernement nazi parvient à mettre au pas la quasi-totalité des Églises protestantes. Le 14 juillet est constituée " l'Église évangélique allemande » (Deutsche Evangelische Kirche, DEK). On peut noter que, parallèlement, le Reichskonkordat entre le Vatican et le Reich est signé le 20 juillet. Dans une allocution radiodiffusée, Hitler s'engage personnellement dans les élections ecclésiastiques qui voient une victoire écrasante des chrétiens allemands. Contre cette marche triomphale vont se développer plusieurs pôles de résistance: dans son ouvrage Theologische Existenz heute (1933) Karl Barth conteste que la transformation politique de l'État suffise pour légitimer une réforme institutionnelle de l'Église. Il exige, aussi bien des jeunes réformateurs que des chrétiens allemands, une réflexion sur le fondement théologique de l'existence de l'Église. 
Cette protestation est suivie de la création par Martin Niemöller du «Pfarrernotbund». Il réagit contre l'introduction du paragraphe aryen dans les Églises prussiennes, introduction que soutenait la faculté d'Erlangen (professeur Paul Althaus) mais que désapprouvait la faculté de Marbourg (Rudolf Bultmann).

Les antagonismes sont exacerbés par l'activisme des chrétiens allemands. Partout où ils ont la majorité, ils travaillent à l'intégration forcée des Église territoriales dans la DEK. En novembre 1933, lors d'un grand rassemblement à Berlin, ils développent une ligne maximaliste demandant d'expurger la Bible de tout relent de judaïsme (Ancien Testament et épîtres de Paul) et de voir en Jésus un héros « aryen ». Cette manifestation suscite la réprobation générale des protestants, le scandale permet à tous les groupes qui refusent la politique centralisatrice de Ludwig Müller d'exprimer leur désaccord théologique. Ces forces d'opposition se regroupent dans l'Église confessante à l'occasion du premier synode confessant de Barmen (31 mai 1934). Il fut précédé par des synodes régionaux et diverses rencontres préalables, notamment à Ulm. On y adopte la Déclaration de Barmen rédigée par Karl Barth. La première et la cinquième thèse du texte de Barth impliquent expressément une condamnation théologique de l'État nazi. L'Église ne peut transformer le contenu de son message ni ses institutions à son gré ou les mettre en accord avec les idées politiques ou philosophiques du temps. L'État a une mission particulière mais ne peut prétendre devenir l'ordre unique et total de toute la vie humaine et exercer ainsi la vocation de l'Église elle-même. Le synode se considère comme le seul représentant théologiquement légitime de la DEK. Sa déclaration veut être l'actualisation des confessions de foi de la Réforme « face aux fausses doctrines des chrétiens allemands et de l'actuel gouvernement de l'Église protestante allemande ».

Dans un premier temps le regroupement des forces confessantes à Barmen n'empêche pas la politique d'intégration forcée menée par l'appareil central de la DEK. Cette politique atteint son paroxysme à l'automne 1934 avec l'arrestation des évêques luthériens de Wurtemberg (Theophil Wurm) et de Bavière (Ludwig Meiser). Ce durcissement provoque la convocation du deuxième synode confessant général à Dahlem, paroisse de Niemöller, les 19 et 20 octobre 1934. Le synode se déclare seul représentant juridiquement légitime de la DEK et revendique pour eux un droit de légitime défense (kirchliches Notrecht) contre les décisions des pouvoirs centraux.

Le 30 octobre, Hitler reçoit les trois évêques luthériens contestataires (Wurm, Meiser et l'évêque de Hanovre, Marahrens) : les mesures législatives qui ont permis l'intégration forcée des Églises territoriales sont abrogées. La politique d'unification et de centralisation forcée des Églises protestantes a définitivement échoué. Après l'échec de Ludwig Müller, l'évêque du Reich, l'État nazi charge Hanns Kerrl du ministère des Affaires ecclésiastiques de parvenir à un consensus avec les Églises protestantes. Cette politique dite de "pacification " provoque une crise au sein de l'Église confessante, les luthériens pour leur part se regroupent au sein du Conseil de l'Église évangélique luthérienne d'Allemagne. La scission de l'Église confessante traduit des divergences ecclésiologiques: les théologiens issus des Églises unies (Kirchen der Union), par exemple l'Église de Prusse (Deutsche Evangelische Kirche der Altpreussischen Union, $\mathrm{APU})$, préconisent une structure congrégationaliste ainsi qu'une Église strictement confessante; les théologiens issus de l'appareil luthérien défendent une conception davantage orientée vers une vision institutionnelle de l'Église et sont prêts à plus de 
compromis avec l'État. L'année 1936 ne marque pas seulement la scission de l'Église confessante. La politique nazie vis-à-vis des Églises commence à s'infléchir. D’une politique d'intégration et de mise au pas, visant à faire des Églises des alliées, on passe à une politique de persécution, cherchant à limiter l'activité et l'influence des Églises. Elle se traduit notamment par l'arrestation d'un certain nombre de pasteurs et de dirigeants de l'Église confessante. Niemöller est le cas le plus célèbre. Elle se traduit aussi par la fermeture de structures de formation théologique, parallèles aux universités, comme le séminaire de Finkenwalde, dirigé par Dietrich Bonhoeffer. Cette Église de « professants » agira donc souvent dans la clandestinité, ses dirigeants furent arrêtés. Niemöller passera huit ans en camp de concentration, Bonhoeffer et d'autres sont exécutés ou envoyés sur le front russe pour des missions sans espoir.

Ce clivage au sein même de l'Église confessante perdure jusqu'à la fin de la guerre et au-delà et explique dans une large mesure les difficultés rencontrées dans l'après-guerre pour organiser le protestantisme allemand au sein d'une nouvelle structure qui s'appellera EKD: Evangelische Kirche in Deutschland (Église évangélique en Allemagne).

\section{L'élaboration de nouvelles structures du protestantisme allemand}

\section{Les conditions}

L'effondrement du Reich sanctionne aussi la faillite des structures de centralisation imposées par la Reichskirche que le régime totalitaire nazi avait en quelque sorte instrumentalisée. La DEK (Deutsche Evangelische Kirche) créée pendant le III Reich est discréditée, non seulement en tant qu'organisation mais aussi en raison des prises de position de ses dirigeants face au nazisme (cas de l'évêque luthérien de Hanovre Marahrens).

Toutes les puissances d'occupation encouragent les mouvements de résistance qui se sont développés et organisés durant le Kirchenkampf. L'Église confessante, malgré les persécutions, a pu se réorganiser et les Alliés reconnaissent son rôle de résistance spirituelle. Ce sont ses cadres qui formeront pour une large part les structures dirigeantes du protestantisme d'après-guerre. L'Église confessante est toujours dirigée par les Conseils de frères (Bruderräte) avec un organisme central, le Conseil des frères du Reich (Reichsbruderrat) créé en 1934. Les luthériens sont regroupés depuis le début de la guerre dans le Conseil des Églises évangéliques luthériennes en Allemagne. On peut noter que ce en Allemagne sera repris par la future dénomination du protestantisme allemand. L'existence de ces deux organismes cache des divergences théologiques et politiques profondes qui n'avaient pas pu être surmontées malgré une lutte commune contre le totalitarisme. Les deux organisations, Conseil des frères de l'Église confessante et Conseil des Églises évangéliques luthériennes, étaient cependant convaincues qu'elles avaient un caractère provisoire déterminé par les conditions de résistance au nazisme et qu'une unification du protestantisme était souhaitable voire impérative. Cette nécessité de s'unifier fut renforcée et précipitée par l'évolution politique de l'aprèsguerre: les risques entraînés par la division de l'Allemagne en zones d'occupation, et la perspective de réorganisation territoriale prévue par les accords de Potsdam, le fait que les frontières artificielles, tracées par les Alliés pour leurs zones d'occupation et les Länder, ne correspondaient en rien aux limites historiques des Églises territoriales - la Prusse et donc l'ancienne Église unie de Prusse est à la fois amputée des territoires 
situés à l'est de la ligne Oder-Neisse et écartelée entre quatre zones d'occupation - et le fait que les frontières entre ces différentes entités territoriales se voulaient imperméables, tout ce contexte politique de l'immédiat après-guerre risquait de paralyser toute action commune des protestants allemands. Il n'y a plus de capitale ni de centre de décision pour coordonner l'action des protestants. Il est donc vital de créer une structure regroupant tous les protestants quelle que soit leur dénomination et quelle que soit la puissance d'occupation dont ils relevaient. Face au vide politique puisqu'il n'y a plus d'État allemand, seule une Église unitaire pourra s'opposer aux tendances centrifuges suscitées éventuellement par l'antagonisme des Alliés et les débuts de la guerre froide. En ce sens le protestantisme est aussi dans l'après-guerre un symbole de l'unité allemande à maintenir, malgré les vicissitudes de l'histoire récente.

Mais cette Église unifiée devait réexaminer ses compromissions et son rapport avec l'État et le nationalisme allemands, elle ne pouvait plus être nationale. La Deutsche Evangelische Kirche (DEK) s'étant définitivement déconsidérée, il fallait tourner la page du nationalisme. Une Église dite « allemande » ne pouvait pas ne pas rappeler les chrétiens allemands. Il n'y aurait donc plus d'Église protestante allemande mais une Église protestante en Allemagne qui quittait définitivement la voie spécifique du protestantisme allemand dans l'histoire pour resserrer les liens avec le réseau développé depuis les années 1920 par le Conseil œcuménique des Églises et se rattacher ainsi au protestantisme mondial.

\section{Réalisation dans l'urgence d'une solution "provisoire " qui se révèlera définitive!}

L'évêque du Wurtemberg, Theophil Wurm, s'attacha à la tâche délicate de réorganiser et de réunir les Églises protestantes en Allemagne. Le 21 août 1945, l'organisme central de l'Église confessante, le Reichsbruderrat, se réunit à Francfort-sur-le-Main et se prononce pour la création d'une structure unique rassemblant et regroupant tout le protestantisme allemand. Fin août, les partisans de ce projet se réunissent à Treysa en Bavière et adressent un appel à l'unité en direction des communautés protestantes et de leurs pasteurs. L'Église muselée, prisonnière voire persécutée sous le III ${ }^{\mathrm{e}}$ Reich, peut désormais, à travers des structures nouvelles, délivrer librement le message de l'Évangile.

Mais cette volonté d'unité paraît bien compromise: derrière la querelle sur l'organisation se cachent des divergences théologiques et ecclésiologiques importantes. Elles portent sur la nature de l'Église, la définition de son rôle dans la société et sa mission dans le monde. Dès ce moment, Martin Niemöller souligne les risques d'une politique de « restauration ». Il faudrait, selon lui, éviter un retour aux Églises territoriales traditionnelles dirigées par un évêque, une structure antérieure au nazisme. Il préconise une plus large initiative des communautés de base et cette primauté des communautés de base doit aussi se traduire dans les nouvelles structures. À cette option s'opposent tous ceux qui veulent conserver les Églises territoriales et éviter un amalgame entre luthériens, réformés et Églises unies, amalgame qui nivellerait les particularismes théologiques. C'est ainsi que l'évêque luthérien de Bavière Meiser propose de créer, à côté de l'EKD, l'Église évangélique luthérienne réunifiée (Vereinigte EvangelischLutherische Kirche Deutschlands, VELKD) afin d'affirmer l'indépendance des Églises territoriales et leur autonomie théologique.

Le 31 août 1945, la conférence de Treysa établit une organisation provisoire des Églises, un Conseil de l'Église évangélique en Allemagne (EKD), mais cette organisation 
unique, tout en ayant un synode annuel commun, ne touche pas aux prérogatives des anciennes Églises territoriales. En même temps fut créée une association d'entraide de l’Église évangélique en Allemagne (Hilfswerk der EKD) dirigée par Eugen Gerstenmaier, indépendante de la Mission intérieure à vocation d'évangélisation. Cet organisme fut bientôt le canal principal de l'aide que les Églises protestantes de l'étranger apportèrent à l'Allemagne détruite et affamée.

En 1947, l'EKD se constitua définitivement lors de la seconde assemblée de Treysa. Elle se définit comme Fédération d'Églises luthériennes, réformées et unies. Ses statuts sont présentés à l'ensemble des Églises membres. On convoque pour juillet 1948 une assemblée constituante (verfassungsgebende Versammlung) qui se réunit à Eisenach et entérine les statuts de l'EKD.

\section{Bilan de cette réunification}

En 1948, l'EKD est en définitive une fédération de 28 Églises territoriales. Les trois dénominations du protestantisme allemand, luthériens, réformés et unis, collaborent au sein de l'EKD. La Fédération est donc constituée par des Églises territoriales qui conservent l'essentiel de leurs prérogatives, mais du point de vue institutionnel, il y a deux grandes innovations: elle est supervisée par un synode et possède une direction unitaire. Les luthériens réunis dans l'Église luthérienne unifiée d'Allemagne (VELKD) y conservent un rôle de premier plan à côté de l'Église évangélique unie. Quant au Conseil des frères de l'Église confessante, il prononce alors sa dissolution, ce qui renforcera la tendance conservatrice au sein de l'EKD. Les craintes de Niemöller se trouvent confirmées, la chance d'un renouvellement des structures du protestantisme allemand n'a pas été exploitée, à un moment où précisément n'existait aucun État allemand qui aurait pu l'infléchir: "la grâce du point zéro » (die Gnade des Nullpunktes), comme le formulera Gustav Heinemann, ne sera pas exploitée.

\section{L'ouverture du protestantisme allemand vers les Églises étrangères et les rapports avec les Alliés occidentaux}

Le 22 juin 1945, l'évêque du Wurtemberg, Theophil Wurm, qui prépare la réorganisation du protestantisme en Allemagne, rencontre à Francfort des représentants des Alliés occidentaux et leur présente les objectifs des Églises protestantes après 1945. Il faut, selon lui, " rechristianiser l'Allemagne ", mais rechristianiser ne signifie pas s'aligner sur les directives de rééducation du peuple allemand selon les normes des Américains car il faut tenir compte des spécificités de l'histoire allemande. Au sein du Comité allié pour les affaires religieuses (ARAC en anglais), on fait droit à ces différences fondamentales de mentalités. La directive JCF 1143 établit le principe de la non-ingérence des vainqueurs dans les affaires ecclésiastiques et religieuses, la réorganisation des Églises - que nous venons de présenter dans le point 2 - incombe aux protestants allemands eux-mêmes.

En juillet 1945, le Conseil œcuménique des Églises ${ }^{(1)}$ délègue un pasteur luthérien américain, Steward W. Herman, pour dresser un tableau du protestantisme allemand.

1 Cet organisme a été préparé par un triple mouvement, missionnaire (Édimbourg 1910), social (Conférence Vie et Action Stockholm 1925) et dogmatique (Conférence Foi et Constitution de Lausanne 
Les rapports de ce pasteur déterminent largement l'image que l'on se fait de ce dernier en Amérique et en Angleterre dans les milieux protestants. À la faveur de ces « inspections " se nouent des contacts et des échanges internationaux fructueux entre responsables d'Église allemands et étrangers. Des laïcs, responsables d'Église, et des pasteurs issus le plus souvent de l'Église confessante, comme Niemöller et Lilje, effectuent des séjours d'études en Scandinavie, Angleterre et États-Unis. Ces figures emblématiques de l'Église confessante puis de l'EKD incarneront à l'étranger pour le monde protestant le nouveau visage du protestantisme allemand et auront même d'importantes responsabilités au sein du Conseil œcuménique des Églises.

Pour conforter cette image positive et réintégrer le protestantisme allemand dans le protestantisme mondial, il convient aussi, à la demande même des Alliés, de procéder à une "épuration " au sein de l'Église. L'évêque de Hanovre Marahrens et le directeur des relations internationales de la DEK, Theodor Heckel, devront quitter leur fonction. Marcel Sturm qui dirige l'aumônerie militaire française en Allemagne demandera à l'Église territoriale du Palatinat de mettre à la retraite son évêque nouvellement nommé, Hans Otto Stichter. Ces cas restent cependant isolés, l'adhésion aux différents organismes nazis n'a pas été le critère de sanction mais uniquement les activités en faveur du nazisme au nom de l'Église. Il faut inscrire ces choix en matière d' " autoépuration » de l'Église protestante dans le cadre plus large du débat sur la culpabilité du peuple allemand que les instances du protestantisme allemand ont engagé courageusement, souvent contre une opinion publique rétive voire hostile.

\section{L'aveu de culpabilité du protestantisme allemand (Stuttgart 18 octobre 1945) devant les représentants du COE}

Si la réorganisation de l'Église protestante allemande après 1945 a été laborieuse et influencée par des instances internationales telles que le COE, il en fut de même pour le débat sur la culpabilité du protestantisme allemand face au nazisme. On peut partir de la déclaration de culpabilité du Conseil de l'EKD faite à Stuttgart le 18 octobre 1945 devant les représentants du Conseil œcuménique des Églises ${ }^{(2)}$.

Le premier fait remarquable est la présence de ces représentants étrangers venus assister au Conseil de l'Église protestante en Allemagne, cette dénomination prend donc tout son sens. Pour la première fois depuis de longues années, les représentants officiels du protestantisme mondial sont présents. Le protestantisme allemand est réintégré dans le protestantisme mondial. Ces représentants du COE viennent de pays qui ont souffert de la guerre, des violents bombardements de la population civile comme l'Angleterre ou de l'occupation allemande brutale et sanglante comme la Norvège et la Hollande. C'est l'archevêque de Canterbury qui répondra personnellement à cette déclaration pour saluer le courage moral qu'implique cette reconnaissance de culpabilité.

1927). Conçu par un comité préparatoire dès 1938, le COE voit le jour dans sa forme institutionnelle le 23 août 1948 à Amsterdam: 147 Églises de 44 pays et de toutes les confessions chrétiennes - à l'exception de l'Église catholique romaine - y sont représentées. Le protestantisme allemand est présent dès l'origine dans les différents comités préparatoires. Martin Niemöller est membre du comité exécutif et central du COE comme directeur des relations internationales.

2 Remarquons que ces deux instances, EKD et COE, n'ont pas encore leur statut définitif en 1945. 
Que dit en substance cette déclaration du Conseil de l'EKD? Nous (les protestants) avec notre peuple, nous sommes solidaires de toutes les souffrances causées par la guerre et avec notre peuple nous sommes solidaires dans la culpabilité. Cette reconnaissance de culpabilité était associée à une réflexion théologique, elle conclut à une faillite de l'Église dans sa mission de prédication pour n’avoir pas été assez courageuse ni assez fidèle à sa vocation dans la confrontation avec le nazisme. L'Église a trahi sa mission, compromise qu'elle était avec un État totalitaire. Cette déclaration a souvent été mal interprétée comme aveu de culpabilité collective (Kollektivschuld) du peuple allemand alors qu'elle se voulait essentiellement manifester sa solidarité dans la culpabilité (Solidarität der Schuld). Elle rencontra relativement peu d'échos dans l'ensemble de la population mais donna lieu à des controverses au sein de l'Église. Le texte autorise en effet plusieurs lectures.

On peut, comme Martin Niemöller et Gustav Heinemann, faire porter l'accent sur la première phrase du texte et envisager une réorientation de tout le peuple allemand, initiée par l'Église protestante. Consciente de sa faute et solidaire des souffrances causées, l'Allemagne peut prendre un nouveau départ. On peut aussi mettre l'accent sur la réconciliation avec les chrétiens dans le monde entier et souligner l'engagement des protestants allemands contre le nazisme tout en admettant son insuffisance et sa faillite. Cet engagement passé permet l'intégration à part entière du protestantisme allemand dans un mouvement mondial. Cette déclaration peut signifier enfin que l'aveu de culpabilité clôt le débat, la question de la culpabilité étant tranchée et le passé assumé, en mettant l'accent uniquement sur le « nouveau départ». On a pu parler dans ce cas d'une minimisation et d'une autojustification plus ou moins manifeste. Au-delà de toute polémique, un fait s'impose: aucune autre Église allemande et aucune institution ayant survécu au nazisme n'a parlé aussi clairement de sa faillite et de sa propre culpabilité face au nazisme ${ }^{(3)}$.

Cet « examen de conscience » sera approfondi en 1947 dans le Darmstädter Wort, un appel et une sorte de testament politique de l'Église confessante. La remise en question du passé protestant ne s'arrête pas au nazisme, il ne peut ignorer les relations que le protestantisme allemand entretient avec l'État et le rapport qui s'est construit à travers les siècles à partir de la théorie des deux Règnes selon Luther. Dans ce texte on fustige: le rêve d'une "vocation allemande " spécifique; le recours sans limite à la force et à la violence; la confiance accordée à un gouvernement fort et autoritaire et la soumission inconditionnelle au Prince souverain et à l'État; l'alliance des Églises avec les puissances conservatrices qui cherchent à maintenir une société inégalitaire; enfin, le rejet catégorique par les Églises de toute action révolutionnaire.

Une nouvelle conception des rapports entre Église et État se dégage de ce texte: l'Église ne peut plus s'identifier à aucun régime politique; même les relations avec la démocratie doivent être critiques. Se référant au texte biblique, l'Église doit assurer un rôle de veilleur, de vigile (Wächteramt). À ce titre, elle s'engage pour tous les hommes quelle que soit la forme de l'État ou du gouvernement. Si peu de dirigeants des Églises protestantes ont signé ce texte radical, beaucoup de mouvements protestants s'en sont réclamés en Allemagne et il continue à servir de référence.

3 On peut comparer ce texte par exemple avec la Lettre pastorale (Hirtenbrief) du 23 août 1945 émanant de la Conférence épiscopale, qui débute par un éloge appuyé du cardinal Bertram. 
Cette réflexion des protestants sur les rapports qu'ils souhaitent établir avec le pouvoir politique nous conduit à plusieurs observations.

Avant même la création de la République fédérale et grâce à leurs relations privilégiées avec le Conseil œcuménique des Églises, les dirigeants de l'EKD étaient fortement impliqués et jouaient un rôle dans les relations internationales avec le monde occidental notamment anglo-saxon, qui débordait largement le cadre strict des Églises. À ce moment, il n'y a pas d'État allemand donc pas de gouvernement central et encore moins de politique étrangère allemande.

Forte des leçons du passé, l'Église protestante nouvelle, dans son ensemble, souhaite maintenir une distance critique vis-à-vis de tout État futur. Elle se veut une communauté au service de l'intérêt général mais son action ne doit pas être soumise aux directives de l'État. À l'inverse, si le chrétien, en vertu de ses convictions religieuses, a un devoir de s'engager en politique, il ne peut le faire au nom de l'institution Église.

Cependant, au sein de l'Église, plusieurs questions continuent à faire débat: que faut-il entendre par Wächteramt? Est-ce le rôle de l'Église de signaler et de combattre les dérives de l'État et les carences de la société? Quel doit être le statut constitutionnel des Églises face à l'État? Dans le cas d'un statut de séparation, peut-il cependant s'établir un " partenariat » entre État et Église? Peut-il y avoir une complémentarité ou une répartition des compétences? Peut-on envisager, même à titre provisoire, un État allemand amputé de l'ancienne zone soviétique, berceau de la Réformation et dont la population est à $80 \%$ protestante? La question nationale place le protestantisme devant des choix déchirants.

Nous verrons en dernière partie comment le protestantisme va s'inscrire dans le nouveau cadre constitutionnel avec la création de la République fédérale d'Allemagne selon la Loi fondamentale (Grundgesetz) en 1949.

\section{Les rapports entre Église et État selon la Loi fondamentale}

Dans cette période d'occupation par les Alliés et de vacance totale d'un État allemand (1945-1949), les Églises catholique et protestantes ont joué un rôle essentiel dans le fonctionnement de la société allemande sur le plan humanitaire, social, culturel mais aussi politique. L'influence et l'autorité des Églises dans la société allemande sont largement reconnues par les Alliés occidentaux qui collaborent avec elles. Elles assurent une continuité dans une société où toutes les autres institutions se délitent. Avec l'aide matérielle et morale de l'étranger, les Églises sont les premières institutions à apporter leur contribution à la refondation d'un État de droit inspiré des démocraties de type occidental. Au-delà de leurs intérêts propres, les Églises plaident la cause des Allemands auprès des Alliés, défendent leurs droits et vont jusqu'à contester certains jugements dans le cadre de la dénazification. Les conflits qui éclatent parfois avec les puissances d'occupation augmentent le prestige des Églises. Celles-ci ont cependant surestimé leur influence et présumé de leur pouvoir, si l'on considère les dispositions relatives à la place et au rôle des Églises dans les constitutions des Länder mais surtout dans l'élaboration de la Loi fondamentale. 


\section{Le statut juridique des Églises selon les différentes constitutions des Länder}

Exception faite de celle de Wurtemberg-Hohenzollern, les dispositions concernant le rapport Église/État dans les nouvelles constitutions des Länder s'inspirent largement de la Constitution de Weimar. Mais selon les majorités politiques en place, les compétences attribuées à l'État sont plus ou moins importantes. La Hesse social-démocrate et protestante accentue la séparation de l'Église et de l'État par rapport à Weimar. En Bavière, Rhénanie-Palatinat, régions à dominante chrétienne-démocrate et catholique, les intérêts du catholicisme sont davantage pris en compte. La plupart des constitutions des Länder reconnaissent les valeurs morales enseignées par les Églises. L'article 41 de la Constitution de Rhénanie-Palatinat reconnaît par exemple leur contribution « à la sauvegarde et au renforcement des bases religieuses et éthiques de la vie humaine».

Les institutions religieuses sont « des corporations de droit public, bénéficiant d'une autonomie de gestion et de droits spécifiques reconnus par la Constitution ». L'école confessionnelle antérieure au nazisme n'est rétablie que dans quelques Länder à forte population catholique, dont la Bavière et la Rhénanie-Palatinat. L'État contracte des engagements financiers vis-à-vis des Églises et prélève, pour leur compte, l'impôt ecclésiastique (Kirchensteuer) qui est inscrit dans toutes les constitutions des Länder. Ce droit a été accordé aux différents Länder par le Conseil interallié en janvier 1946.

En revanche, la Loi fondamentale fut élaborée dans un contexte peu favorable aux revendications des Églises, catholique en particulier. Il ne crée pas un système vraiment nouveau dans les rapports entre Église et État, mais par l'article 140, il reprend, dans l'improvisation et comme solution de fortune, les dispositions de la Constitution de Weimar. Il les modifie cependant au sens où il transfère aux Länder toute compétence pour leur interprétation et leur application.

\section{Les demandes des Églises au Conseil parlementaire}

Le $1^{\text {er }}$ septembre 1948, le Conseil parlementaire (Parlamentarischer Rat) se réunit à l'initiative des Alliés occidentaux et sous leur contrôle. Il travaille sur la base de projets élaborés à Herrenchiemsee en août 1948. Nous n’abordons ici que les dispositions concernant l'Église et l'État. Le Conseil dispose d'une lettre pastorale (Hirtenbrief) où l'Église catholique à travers le collège de ses évêques formule ses demandes. Elle s'attend à ce que l'État soit « constitué et institué selon les plans de Dieu ». Cela implique pour les évêques catholiques l'inscription dans la constitution des droits imprescriptibles de la personne, la protection de la famille, le caractère sacré du mariage, le droit à la vie de tout enfant dès sa conception, et un droit naturel des parents à l'éducation de leurs enfants.

Étant donné les fluctuations de majorité dans cette assemblée composite, il était essentiel pour les Églises d'établir des contacts réguliers avec les membres de cette assemblée. Le prélat Wilhelm Böhler, conseiller politique du cardinal Frings de Cologne, était le représentant officiel de l'Église catholique, chargé de coordonner ce travail de prise de contact avec les membres du Conseil parlementaire. Son rôle a été très actif et il incarne l'efficacité politique du catholicisme allemand dans cette phase de construction de la République fédérale. Cependant et bien que Böhler soit parvenu à gagner à sa cause la majorité du groupe parlementaire chrétien-démocrate, le Conseil parlementaire n'a pas repris à son compte l'ensemble de ses demandes et a 
préféré mettre entre parenthèses la question des rapports de l'Église et de l'État. Le Conseil a évité ainsi une politisation et une confessionnalisation du débat, les questions constitutionnelles ne relevant pas d'une approche religieuse et confessionnelle. Néanmoins l'Église catholique organisa une action concertée, sous forme de requête aux parlementaires, où elle a pu associer l'Église protestante de Rhénanie et celle de Westphalie sur la question scolaire. En accord avec le Vicariat général de Cologne, les Églises protestantes de Rhénanie et de Westphalie ont revendiqué « le droit parental à déterminer l'éducation de leurs enfants ", c'est-à-dire implicitement l'institution d'écoles confessionnelles.

Le 9 novembre 1948, le président de l'EKD, Theophil Wurm, lance l'avertissement suivant: "Si le Conseil parlementaire veut éviter des difficultés et des déconvenues, il doit au préalable prendre contact avec les Églises en vue de fixer les dispositions fondamentales sur les rapports de l'Église et de l'État. » Wurm dit expressément qu'il faudra revenir sur l'ensemble de la question, il affirme que l'enseignement religieux doit être introduit dans toutes les écoles comme matière obligatoire à part entière, et que le droit parental doit être garanti par la Constitution. Il demande en outre la protection de l'enfant dès sa conception, l'interdiction de la stérilisation des personnes et de toute expérimentation sur des personnes vivantes. Par ces demandes, Wurm revendique pour son Église, comme il le souligne expressément, non seulement un droit de co-décision en matière de législation sur les Églises mais aussi une compétence et une responsabilité éthique dans la société. Les exemples qu'il cite rappellent directement les atteintes aux droits de la personne sous le nazisme et les protestations qu'elles avaient alors suscitées au sein des Églises catholique et protestantes. Les critiques ont fait valoir, à bon droit sans doute, que cette position de l'EKD privilégiait la responsabilité éthique et que le débat sur la nature des relations entre Églises et État passait au second plan.

Le Conseil de l'EKD, constitué après 1945, se réunit pour la dernière fois les 12 et 13 décembre 1948. Il aborde la question des «positions de l'Église vis-à-vis de la forme de gouvernement ». Il charge Martin Niemöller et Heinrich Held, président de l'Église de Rhénanie, secondés dans leur tâche par la chancellerie de l'EKD, de présenter une requête au Conseil parlementaire. Le procès-verbal dit en substance que l'Église doit présenter des revendications sur lesquelles elle ne pourra transiger même si ses demandes pouvaient susciter des tensions au sein du Conseil parlementaire. Cette requête pourtant ne fut jamais présentée. Martin Niemöller prend de nouvelles fonctions, cette fois au sein de l'ACK (Arbeitsgemeinschaft Christlicher Kirchen in Deutschland) qui à la différence de l'EKD inclut les Églises protestantes libres - et c'est à ce titre et au nom de l'ACK qu'il va s'adresser au Conseil parlementaire. Il signale que l'Église protestante au sens large porte le plus grand intérêt non seulement aux réglementations concernant directement les Églises mais aussi à tout cadre institutionnel garantissant les droits de l'homme et de la personne. En outre il demande l'introduction dans la Constitution d'un article spécifique garantissant aux Églises chrétiennes de diffuser directement leur message non seulement dans le cadre cultuel mais aussi en dehors de ce cadre, sans doute songe-t-il à l'article 149 de la Constitution de Weimar qui accordait ce droit aux Églises. En conclusion, il demande pour les Églises un droit inconditionnel de prendre position sur tous les problèmes de société. Selon Niemöller, l'Église a un message à transmettre, une mission à accomplir, elle est au service du 
peuple allemand, mais aussi de tous les peuples et cette mission ne saurait être entravée par des dispositions légales. Heinrich Held, le président de l'Église protestante de Rhénanie, désigné par l'EKD pour défendre les intérêts du protestantisme au sein du Conseil parlementaire, ne s'est guère distingué dans les débats. Sur la question scolaire, c'est même le prélat de Cologne Böhler qui a défendu la cause commune des catholiques et des protestants.

\section{Réactions et stratégie du Conseil parlementaire face aux requêtes des Églises protestantes}

Le Conseil parlementaire travaillait dans une situation d'urgence à la rédaction de la nouvelle Constitution et les questions religieuses ne représentaient pas pour lui une priorité. Pour éviter un examen approfondi et une redéfinition des rapports entre l'État et les Églises, le Conseil parlementaire est très vite revenu aux dispositions adoptées en 1919 par la Constitution de Weimar et qui, en instaurant une séparation de l'Église et de l'État, étaient novatrices à l'époque. On mettait entre parenthèses la politique ambiguë des Églises vis-à-vis du nazisme, voire leur faillite, pour renouer avec les termes mêmes de la Constitution de Weimar. Néanmoins font problème, d'une part l'unité forcée des Églises protestantes imposée par les nazis avec la création en 1933 de la Reichskirche et d'autre part le Reichskonkordat signé par le III ${ }^{\text {e }}$ Reich totalitaire avec le Vatican. La Reichskirche a disparu dans ses structures centralisées avec l'effondrement du III Reich. Que va-t-il advenir du Concordat signé par cet État totalitaire? Les protestants sont hostiles au maintien de ce Concordat. Le SPD tout comme le FDP engagent une polémique contre le maintien de cet accord. Adolf Süsterhenn, catholique, membre de la CDU, souhaite à la fois la reprise des dispositions de la Constitution de Weimar en matière religieuse et le maintien du Concordat. Theodor Heuss (FDP) n'accepte finalement la proposition de Süsterhenn de reprendre dans la Loi fondamentale les dispositions de Weimar qu'à condition de transférer toute souveraineté en matière religieuse aux Länder qui ont à légiférer, chacun pour sa part, sur l'avenir du Concordat. L'article $123 \mathrm{du}$ Grundgesetz stipule que même un traité signé entre deux États, ici le Vatican et le Reich, doit être réexaminé dans le cadre de la législation de chaque Land.

Une autre controverse importante se développe autour de la formulation de la Constitution de Weimar: "Il n'existe pas d'Église d'État [Staatskirche] ». Cette disposition est maintenue dans la Loi fondamentale, elle implique qu'on ne parle plus désormais d'un statut spécifique des Églises, mais du statut des sociétés religieuses (Religionsgesellschaften) dont le statut juridique ne diffère pas de celles qui se fondent sur une idéologie, une vision de l'univers (Weltanschauungsgesellschaften). L'article 140 de la Loi fondamentale qui correspond aux articles 136 et suivants de la Constitution de Weimar établit ainsi une parité entre sociétés religieuses et autres sociétés de pensée. Les conservateurs ne sont pas arrivés à amender ce texte en imposant dans la Loi fondamentale une distinction entre Églises et Religionsgemeinschaft (communauté fondée sur la religion), une distinction qui aurait assuré un statut privilégié aux Églises.

Pourquoi les dispositions de la Loi fondamentale ne reflètent-elles pas davantage l'influence que les Églises avaient acquise entre 1945 et 1949? Le Conseil parlementaire fonctionne selon les principes du consensus politique, comme le rappelle Theodor Heuss dans un discours de janvier 1949. Ce rapport consensuel semble gravement 
compromis lorsque la CDU réclame que des dispositions particulières sur la famille, l'éducation et l'école figurent dans la Loi fondamentale. Or les sociaux-démocrates (SPD) ont accepté, dans un esprit de conciliation, que ni le droit de grève ni le droit au travail réclamé par le chef du DGB, Hans Böckler, ne figurent dans la Constitution. En contrepartie, les sociaux-démocrates obtiennent des députés conservateurs (CDU/ CSU) le rejet de toutes les demandes de l'Église catholique concernant le droit parental à l'éducation des enfants dans des écoles confessionnelles. Dans la Constitution de 1949 ne figurent donc ni la généralisation de l'école confessionnelle, ni surtout un statut particulier des Églises.

Sur toutes ces questions, le FDP soutient le camp du SPD. Malgré un afflux de protestations et d'appels émanant de l'Église catholique, des associations de parents et des paroisses catholiques, et même d'une intervention directe du pape Pie XII dans une lettre adressée aux cardinaux allemands, ces demandes catholiques seront rejetées.

Malgré la violence de ces différends idéologiques, le bon déroulement des travaux parlementaires n'a jamais été entravé. La volonté de conclure par un compromis, dans le domaine religieux également, l'a toujours emporté au sein de ce Conseil sur les divergences d'opinion grâce aux leaders politiques tels que Th. Heuss (FDP), K. Adenauer (CDU) et Carlo Schmid (SPD), plus soucieux de l'intérêt du peuple allemand que des intérêts particuliers des Églises. Carlo Schmid rend hommage à l'attitude d'Adenauer : «Il était moins clérical qu’on ne pense. Lorsque nous avons annoncé au prélat Böhler que l'accord s'était fait [au sein du Conseil parlementaire] sur les questions religieuses, celui-ci a annoncé que jamais l'Église ne dirait oui à une telle décision. Adenauer répliqua alors à Mgr Böhler: "Dans ces affaires, on ne demande pas à l'Église de dire oui ou non, mais de dire tout au plus amen.” ”

\section{L'accueil mitigé des Églises}

La Loi fondamentale est promulguée le 23 mai 1949. L'épiscopat allemand prend position dans une déclaration officielle de mai 1949 où il est extrêmement critique. Il relève d'abord une grave lacune: l'absence de dispositions sur la question de l'école confessionnelle et sur le droit des parents à décider de l'éducation religieuse des enfants. Pour l'Église catholique, ce droit des parents fait partie des droits fondamentaux.

Certains milieux protestants émettent également des critiques. Certaines relèvent de la polémique: Niemöller par exemple n’a que méfiance vis-à-vis de ce nouvel État, " conçu au Vatican et enfanté à Washington " (vom Vatikan gezeugt und in Washington geboren). D’autres critiques paraissent plus fondées. Cet État dit «État occidental » et sa Loi fondamentale ne sauraient constituer qu'une solution provisoire. Les protestants, majoritaires à l'Est comme à l'Ouest, sont attachés à l'unité de la nation - or, la Loi fondamentale encourage la division de l'Allemagne par ses choix unilatéraux et la scission se fait au détriment du protestantisme majoritaire dans la zone soviétique. Un État uniquement tourné vers l'Occident sacrifie l'unité du peuple allemand. C'est pourquoi le Conseil de l'EKD déclare le 12 octobre 1949 à propos de la situation politique issue de la création successive de la RFA puis de la RDA: "Malgré toutes les décisions des puissances temporelles [weltliche Mächte] qui nous gouvernent, nous demeurons un seul peuple et nous avons l'obligation de vivre en communauté fraternelle et dans le respect mutuel qui convient à des frères. " La tentation d'une neutralisation de 
l'Allemagne s'affirme alors chez certains protestants comme M. Niemöller et G. Heinemann pour maintenir et conforter ce lien, le protestantisme constituant un pont entre l'Est et l'Ouest.

\section{Conclusion}

Le protestantisme allemand n'avait pas rallié la République de Weimar. Nostalgique qu'il était de son statut de « religion d'État », en Prusse par exemple, il avait combattu le nouveau statut de séparation de l'Église et de l'État. La logique du Kirchenkampfl'avait conduit à l'époque du totalitarisme nazi, non seulement à se méfier de l'État mais à le défier et à s'en émanciper. Par la confession de Barmen de 1934, le protestantisme s'est transformé d'une Église multitudiniste (Volkskirche) en une Église confessante, une Église de professants. Dans le souci de rendre crédible son témoignage chrétien, elle remet fondamentalement en question sa conception de l'État et de ses droits. Par la déclaration de Stuttgart sur la culpabilité (Schuldbekenntnis) et par celle de Darmstadt sur les pesanteurs historiques du protestantisme allemand depuis Luther, elle en appelle à une vigilance critique vis-à-vis de la politique et veut assumer une fonction de «veilleur» face au monde politique (Wächteramt). En 1949, le protestantisme ne peut que souhaiter un statut de séparation de l'Église et de l'État. La reprise presque in extenso des textes constitutionnels de Weimar semble suggérer un retour à la situation de 1919. Mais ce régime de séparation que les protestants avaient combattu durant le régime de Weimar, ils l'acceptent en 1949 sans difficulté et sans réserve.

Le souci de l'unité organique du protestantisme constitue la seconde originalité de cette période. À Treysa en août 1945, les protestants affirment pour la première fois leur volonté d'unité et définissent les structures de l'EKD, Église protestante en Allemagne, une unité de type fédéral, dans le respect des appartenances confessionnelles et historiques. Ils se réunissent dans une structure unique et indépendante des Länder. Les statuts de l'EKD sont adoptés à Eisenach - ce haut lieu de la Réforme avec la Wartburg - le 13 juillet 1948, c'est-à-dire exactement 15 ans après la création sous le nazisme de la Reichskirche. Mais dès sa constitution, cette structure nouvelle sera fragilisée par les débuts de la guerre froide puis la division de l'Allemagne. La dénomination d'Église protestante en Allemagne signifie aussi le refus d'une division de l'Allemagne.

La séparation de l'Église et de l'État sur le modèle de Weimar va changer de nature et de signification en 1949. Ce n'est plus le rapport à l'État qui fait problème, les protestants ne peuvent que se féliciter d'une neutralité de l'État à leur égard, ainsi que de l'autonomie et de la liberté qui en découlent. Ce qui inquiète en revanche les héritiers de l'Église confessante qui dirigent l'EKD, ce sont les choix politiques du futur État. Selon Niemöller, Heinemann et beaucoup d'autres, la RFA fait un choix dangereux et sans retour en optant avec Adenauer résolument pour l'Occident et en préparant le réarmement. Les protestants de l'Est sont les victimes de cette politique. Pendant des années, l'EKD engage une politique de neutralité face aux deux blocs qui s'opposent dans la guerre froide et tente de combler le fossé qui se creuse entre l'Est et l'Ouest, en maintenant cette structure de l'EKD qui rassemble les protestants des deux Allemagnes. Le débat ne porte plus sur le statut de l'Église par rapport à l'État, tel que le définit la Loi fondamentale, mais sur la nature du nouvel État - réputé précaire et inachevé - ainsi que sur ses choix politiques. 


\section{Bibliographie}

Gerhard Besier, Jörg Thierfelder, Ralf Tyra (dir.), Kirche nach der Kapitulation, vol. 1: Das Jahr 1945 - eine Dokumentation, Stuttgart, Kohlhammer, 1989, 310 p.

Armin Boyens, "Das Stuttgarter Schuldbekenntnis vom 19. Oktober 1945. Entstehung und Bedeutung ", Vierteljahreshefte für Zeitgeschichte, 19 (1971), p. 374-397 (disponible sur Internet en pdf).

Heinz Brunotte, Die Evangelische Kirche in Deutschland. Geschichte, Organisation und Gestalt der EKD, Gütersloh, Mohn, 1964, 208 p.

Martin Greschat, « Kirche und Öffentlichkeit in der deutschen Nachkriegszeit (1945-1949) », in: Kirchen in der Nachkriegszeit. Vier zeitgeschichtliche Beiträge, Göttingen, Vandenhoeck \& Ruprecht, 1979, p. 100-124.

Jürgen HaCH, Gesellschaft und Religion in der Bundesrepublik Deutschland: eine Einführung in die Religionssoziologie, Heidelberg, Quelle \& Meyer, 1980, 217 p.

Frédéric Hartweg, « Gustav Heinemann, ein Christ in der politischen Verantwortung », Revue d'Allemagne, t. X (1978), nº 1 (p. 109-121), 3 (p. 404-457) et 4 (p. 584-617).

Günther Heidtmann, Hat die Kirche geschwiegen? Das öffentliche Wort der evangelischen Kirchen in den Jahren 1945-1964, Berlin, Lettner-Verlag, 1964 (3éd.).

Stewart W. Herman, The Rebirth of the German Church, New York/Londres, Harper \& Brothers, 1946.

Hans-Wolfgang HeßLer (dir.), Protestanten und Kirche in der Bundesrepublik Deutschland, Munich/Vienne, Olzog, 1976, 375 p.

Wolfgang Huber, Kirche und Öffentlichkeit, Stuttgart, Klett, 1973, 736 p.

Werner Jochmann, « Evangelische Kirche und politische Neuorientierung in Deutschland 1945 ", in: Deutschland in der Weltpolitik des 19. und 20. Jahrhunderts. Festschrift für Fritz Fischer, Düsseldorf, Bertelsmann-Universitätsverlag, 1973, p. 545-562.

Heinz KLoppenburg, « Die evangelische Kirche von 1945 bis 1970 », in: Karl-Dietrich BRACHER, Nach 25 Jahren. Eine Deutschlandbilanz, Munich, Kindler, 1970, p. 353-369.

Dieter Koch, Heinemann und die Deutschland-Frage, Munich, Kaiser, 1972, 570 p.

Bernd W. KuввIG, Kirche und Kriegsdienstverweigerung in der BRD, Stuttgart, Kohlhammer, 1974, $128 \mathrm{p}$.

Friedrich Merzyn (dir.), Kundgebungen: Worte und Erklärungen der Evangelischen Kirche in Deutschland (1945-1959), Hanovre, Verl. des Amtsblattes der Evangelischen Kirche in Deutschland, 1960, $352 \mathrm{p}$.

Jürgen Moltmann (dir.), Bekennende Kirche wagen: Barmen 1934-1984, Munich, Kaiser, 1984, $273 \mathrm{p}$.

Annemarie Smith-von Osten, Von Treysa 1945 bis Eisenach 1948: Zur Geschichte der Grundordnung der Evangelischen Kirche in Deutschland, Göttingen, Vandenhoeck \& Ruprecht (Arbeiten zur Kirchlichen Zeitgeschichte, Reihe B-Darstellungen), 1980.

Frederic Sроттs, Kirchen und Politik in Deutschland, Stuttgart, Deutsche Verlagsanstalt, 1976, $358 \mathrm{p}$.

Klaus Steuber, Militärseelsorge in der Bundesrepublik Deutschland. Eine Untersuchung zum Verhältnis von Staat und Kirche, Mayence, Matthias-Grünewald-Verlag, 1972, 226 p.

Johanna Vogel, Kirche und Wiederbewaffnung. Die Haltung der Evangelischen Kirche in Deutschland in den Auseinandersetzungen um die Wiederbewaffnung in der Bundesrepublik 1949-1956, Göttingen, Vandenhoeck \& Ruprecht, 1978, 304 p.

Theophil Wurm, Erinnerungen aus meinem Leben, Stuttgart, Quell-Verlag, 1953, 222 p. 


\section{Résumé}

Pour comprendre l'évolution du rapport à l'État du protestantisme allemand et son acceptation en 1949 du régime de séparation Église/État, il faut revenir notamment sur le Kirchenkampf et ses répercussions. La réorientation et mutation qui s'engage en 1945 comprend l'affirmation d'une solidarité dans la culpabilité, la volonté de créer une structure ecclésiale unique face au vide politique, un réexamen radical du rapport à l'État et au nationalisme. Face au Conseil parlementaire, les deux Églises catholique et protestante sont engagées dans des revendications (codécision en matière de législation et responsabilité éthique, du côté protestant) et tractations diverses (inscription du droit des parents à décider de l'éducation religieuse de leurs enfants, du côté catholique) qui n'aboutissent pas. Si les protestants se satisfont du transfert aux Länder des compétences en matière d'interprétation et d'application des dispositions constitutionnelles relatives aux Églises, ils sont plus réservés sur la question nationale, puis sur les choix politiques effectués, tel l'ancrage à l'Ouest ou le réarmement.

\section{Zusammenfassung}

Um dem Wandel des Verhältnisses zwischen Staat und Protestantismus in Deutschland nach 1945 und der Hinnahme der Trennung von Kirche und Staat im Grundgesetz gerecht zu werden, kann eine Rückschau auf den Kirchenkampf im Dritten Reich und dessen Auswirkungen hilfreich sein. Schwerpunkte der nach 1945 erfolgten neuen Ausrichtung der evangelischen Kirche in Deutschland sind die Solidarität in der Schuld, eine radikale Aufarbeitung des Verhältnisses zum Staat und zum Nationalismus und das Bestreben, angesichts des politischen Vakuums eine einheitliche Kirchenstruktur zu schaffen. Dem Parlamentarischen Rat gegenüber stellten die Protestanten Forderungen (Mitbestimmung, Einflußnahme im gesetzgeberischen Bereich) auf, denen nicht stattgegeben wurde. Die EKD fand sich zwar damit ab, dass die Zuständigkeit in der Auslegung und Umsetzung der staatskirchenrechtlichen Verfassungsbestimmungen nun an die Länder übertragen wurde, sie blieb jedoch gespalten und zurückhaltend hinsichtlich der Nationalen Frage und mancher politischen Weichenstellungen (Westintegration, Wiederaufrüstung). 